\title{
Empirical Study on Stereotype for Burner-Control Relationship of Four-Stove Gas Range for Koreans
}

\author{
Dohyung Kee \\ Department of Industrial and Management Engineering, Keimyung University, Daegu 704-701
}

\begin{abstract}
Objective: This study aims to empirically investigate population stereotype of burner-control linkage of four-stove gas range for Koreans. Background: The previous studies' results for gas range stereotypes were different depending upon methods adopted, i.e., whether using questionnaires, computer simulation or physical models. It is known that the physical model experiment should not be methodologically replaced by the computer simulation or paper-and-pencil tests. Stereotype of gas range for Koreans was surveyed based on questionnaires, but has not been dealt with by using physical models. Method: An experiment was conducted to investigate stereotype of four-burner gas range, in which 32 subjects participated and a real gas range available in the market was bought and used. Four types of burner-control linkage were used as independent variable, and reaction time as dependent variable. Results: ANOVA revealed that four types of burner-control linkage and subjects' gender were not significant on reaction time. Duncan's multiple range test showed that reaction times for type III was significantly lower than those for the other three types of burner-control linkage $(\alpha=0.05)$. Conclusion: It is concluded based on the results of this study that stereotype of gas range for Koreans is type III. This is in agreement with results of existing studies using questionnaire survey, while different from those based on physical models. Application: The results of this study would be useful as an ergonomic guideline when designing gas ranges or similar equipments for minimizing operation errors.
\end{abstract}

Keywords: Affective design, Wide LCD monitor, Frame size, Shape preferences, MDS analysis

\section{Introduction}

양립성은 인간의 기대와 모순되지 않는 자극들 간, 반응들 간 혹은 자극-반응 조합과의 관계를 말한다. 양립성은 크게 개념, 운동, 공간 및 양태(modality) 양립성으로 분류된다 (Kee et al., 2010). 양립성에 대한 연구로는 4 구 가스레인 지의 버너-조종장치 간의 연결 관계가 가장 많이 다루어져 왔다. Chapanis와 Lindenbaum(1959)은 나무로 된 3가지 형태의 가스레인지 모형을 대상으로 평가한 결과 Type II (Table 1 참조)가 조작 에러가 가장 적음을 밝혔다. 시중에
판매되고 있는 가스레인지 조사를 바탕으로 4 가지 형태의 버너-조종장치 연결 형태에 대한 실험을 한 Ray와 Ray (1979)의 연구에서도 Chapanis와 Lindenbaum(1959)와 같이 Type II의 에러율이 가장 낮았다. 설문조사를 이용하여 가스레인지 스테레오타이프를 조사한 연구(Hsu and Peng, 1993; Shinar and Acton, 1978; Wu, 1997)에서는 Type III가 가장 선호되는 것으로 나타났다. 중국인을 대상으로 모형을 이용한 $\mathrm{Wu}(1997)$ 의 연구에서는 기존 연구와 달리 반응시간과 에러율 관점에서 Type $\mathrm{V}$ 가 가장 우수한 것으로 나타나 연구 방법에 따라 결과가 달랐다. 미국인을 대상으로 한 연구(Chapanis and Lindenbaum, 1959; Shinar and

Corresponding Author: Dohyung Kee. Department of Industrial and Management Engineering, Keimyung University, Daegu 704-701.

Mobile: +82-10-5656-1260, E-mail: dhkee@kmu.ac.kr

Copyright@2012 by Ergonomics Society of Korea(pISSN:1229-1684 eISSN:2093-8462 http://www.esk.or.kr). All right reserved.

(c) This is an open-access article distributed under the terms of the Creative Commons Attribution Non-Commercial License(http://creativecommons.org/licenses/by-nc/3.0/), which permits unrestricted non-commercial use, distribution, and reproduction in any medium, provided the original work is properly cited. 
Acton, 1978; Ray and Ray, 1979)에서는 Type II 혹은 III가, 중국인을 대상으로 하는 연구(Hsu and Peng, 1993; $\mathrm{Wu}, 1997$ )에서는 Type III 혹은 V가 선호되거나 에러율이 낮거나 반응시간이 짧게 나타나, 민족 간에 스테레오타이프 에 차이가 있음을 보였다. 이는 스테레오타이프가 사용자 배 경 및 문화에 따라 달라질 수 있다(Hsu and Peng, 1993; Kroemer et al., 1994)는 사실을 보인 것이라 할 수 있다.

2 차원 조종장치-표시장치 배열에서는 설문조사를 이용한 연구와 모형을 이용한 스테레오타이프 연구 결과가 잘 일치 하는 것으로 알려져 있다. 그러나 3차원 배열의 스테레오타 이프는 설문조사보다는 모형이나 실물을 이용하여야 신뢰할 수 있는 결과를 얻을 수 있다(Hoffman, 2009).

한국인을 대상으로 한 연구(Kee, 2011a,b)에서는 설문조 사를 이용하여 Type III가 가장 선호되는 것으로 나타났다. 앞서 살펴본 바와 같이 가스레인지 버너-조종장치 연결 형 태에 대한 스테레오타이프가 연구 방법, 민족에 따라 다름 을 알 수 있다. 가스레인지 버너-조종장치 연결 형태는 3 차 원으로 설문조사보다는 모형이나 실물을 이용한 연구가 좀 더 신뢰성 있는 스테레오타이프를 제시할 것으로 기대되나 (Hoffman, 2009), 이에 대한 한국인을 대상으로 하는 연구 는 없는 실정이다.

따라서 본 연구에서는 한국인의 버너-조종장치 연결 형태 에 대한 스테레오타이프를 실물 가스레인지를 이용한 실험 을 통하여 제시하고, 설문조사를 이용한 연구 결과와 비교하 고자 한다.

\section{Method}

\subsection{Subjects}

본 연구의 가스레인지 스테레오타이프 조사 실험에는 32 명의 대학생 (남 20명, 여 12명)이 참여하였다. 피실험자 의 연령, 신장 및 체중은 각각 $21.94 \pm 2.40$ 세, $170.50 \pm$ $8.00 \mathrm{~cm}, 64.03 \pm 12.10 \mathrm{~kg}$ 이었다. 모든 피실험자는 가스레 인지 조작에 사용되는 팔, 손가락 관련 근골격계질환을 않은 병력이 없는 건강한 대학생이었다. 피실험자에게는 실험 참 여 수당을 지급하였다.

\subsection{Experimental apparatus}

본 연구의 실험에는 가스레인지 $(\mathrm{GRA}-41 \mathrm{Wg}$, 동양매직 (주)), PC(Magic Station, 삼성전자(주)), LabView DAQ (data acquisition) system(cDAQ-9178, NI Instruments) 이 사용되었다. 가스레인지와 PC와의 인터페이스는 LabView
$\mathrm{DAQ}$ system을 이용하여 구현하였다(Figure 1). 모든 실험 과정은 LabView로 구현된 프로그램을 통하여 $\mathrm{PC}$ 로 통제되 며, 실험 결과는 모두 $\mathrm{PC}$ 에 자동으로 저장되도록 하였다.

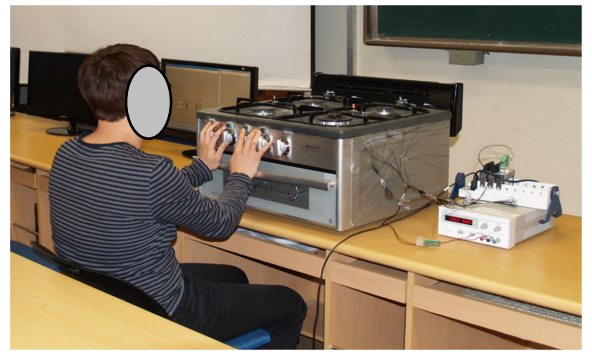

Figure 1. Experimental layout

\subsection{Experimental design}

본 연구에서는 4 구 가스레인지의 버너-조종장치의 가능한 배열 16가지(4!) 중, 기존 연구(Shiner and Acton, 1978; Hsu and Peng, 1993; Wu, 1997; Ray and Ray, 1979)에 서와 같이 4 가지 배열을 사용하였다. 사용된 4 가지 배열은 Figure 2에서와 같이 버너를 알파벳 문자로 표기할 때, 각 버너와 연결된 조종장치의 순서가 왼쪽에서부터 $\mathrm{ABDC}$, $\mathrm{ABCD}, \mathrm{BADC}, \mathrm{BACD}$ 인 형태이다(Table 1). 기존 연구와 의 비교를 위하여 Chapanis와 Lindenbaum(1959), Ray와 Ray (1979) 가 사용한 명칭 (Type II, III, IV, V)을 그대로 사 용하였다.

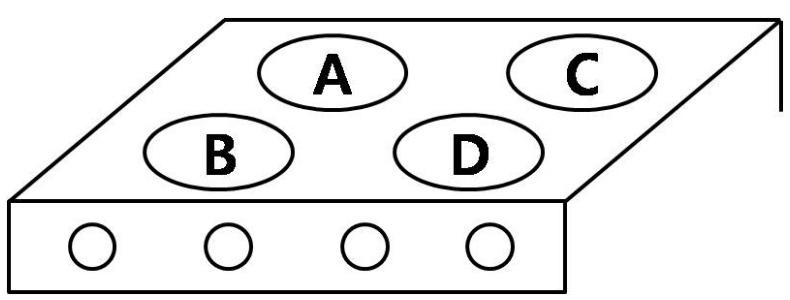

Figure 2. Schematic representation of the gas range burner and control designs

Table 1. Control-burner arrangement relationship

\begin{tabular}{c|c}
\hline Type & Assigned control order \\
\hline II & ABDC \\
\hline III & ABCD \\
\hline IV & BADC \\
\hline V & BACD \\
\hline
\end{tabular}


본 연구의 실험 프로토콜(protocol)은 Chapanis와 Lindenbaum(1959), Hsu와 Peng(1993), Wu(1997)의 연 구를 따랐다. 실험에서 독립변수는 앞서 언급한 4가지 버너 -조종장치 연결 형태이며, 종속변수는 올바른 조종장치를 누르는 데까지 소요된 반응시간이다. 실험에서 4 개의 버너 중 임의의 버너에 빨간 등이 켜지면 피실험자는 가능한 빠르 게 적절한 버튼을 눌러 등을 끄도록 하였다. 만약 피실험자 가 잘못된 조종장치를 누르면 빨간 등은 꺼지지 않으며, 올 바른 조종장치를 눌러 등이 꺼질 때까지 피실험자는 실험을 반복하게 된다. 반응시간은 임의의 버너 등에 불이 들어올 때부터 피실험자가 올바른 조종장치를 눌러 등이 꺼질 때까 지의 시간으로 정의되었다.

실험은 각 배열 형태(Type II, III, IV, V) 별로 하루씩 총 4 일에 걸쳐 실시되고, 4 개의 버너에 대해 각 20회씩 총 80회의 실험이 수행되었다. 4 가지 배열 형태에 대한 실험 순 서는 피실험자별로 임의로 제시되었다. 배열 형태별 실험은 기억 효과를 최소화하기 위하여 실험 간에 최소 2 일의 기간 을 주었다. 실험에서 각 버너에 등이 켜지는 순서도 프로그 램을 통하여 임의로 제시되었다. 버너 등이 꺼진 다음 켜질 때까지의 간격은 1 초로 하였다.

\subsection{Experimental procedure}

실험 절차는 다음과 같다. 먼저 실험을 시작하기 전에 피 실험자에게 실험의 목적 및 내용을 설명하고, 실험 참여 동 의서를 받았다. 피실험자가 동의하면 주어진 배열 형태에 대 한 연습을 10 회 이상 실시하였다. 다음은 본(main) 실험을 정하여진 실험 프로토콜에 따라 80회 실시한다. 본 실험이 2 분 이내의 짧은 시간이 소요되기 때문에 실험 중에는 휴식 시간이 주어지지 않았다. 실험 시작 후의 전 과정은 컴퓨터 로 통제되며, 반응시간은 자동으로 컴퓨터에 파일로 기록 된다.

\section{Results}

\subsection{ANOVA}

본 연구에서 설정한 독립변수인 4 가지 배열 형태와 피실 험자 성별이 반응시간에 유의한 영향을 미치는지를 알아보 기 위하여 분산분석을 실시하였다. 분산분석 결과는 Table 2에 정리되어 있다. Table 2에서 보는 바와 같이 요인으로 잡은 배열 형태, 성별 및 교호작용 모두 반응시간에 통계적 으로 유의한 영향을 미치지 않음을 알 수 있다.
Table 2. Results of ANOVA

\begin{tabular}{c|c|c|c|c}
\hline Source & DF & Sum of squares & $F$ value & $P$ value \\
\hline Type & 3 & 0.1344 & 1.53 & 0.209 \\
\hline Sex & 1 & 0.0062 & 0.21 & 0.645 \\
\hline Type*sex & 3 & 0.0486 & 0.56 & 0.646 \\
\hline Error & 120 & 3.5088 & & \\
\hline
\end{tabular}

\subsection{Effect of arrangement type}

버너-조종장치 연결 형태에 따른 평균 반응시간이 Figure 3 에 나와 있다. 반응시간은 Type III가 가장 짧고, 다음으로 Type V, Type II, Type IV 순으로 커짐을 보였다. Duncan 의 다중범위 검정 (multiple range test)에서 Type III가 다 른 세 가지 유형에 비하여 유의수준 $5 \%$ 에서 반응시간이 유 의하게 작은 것으로 나타났다.

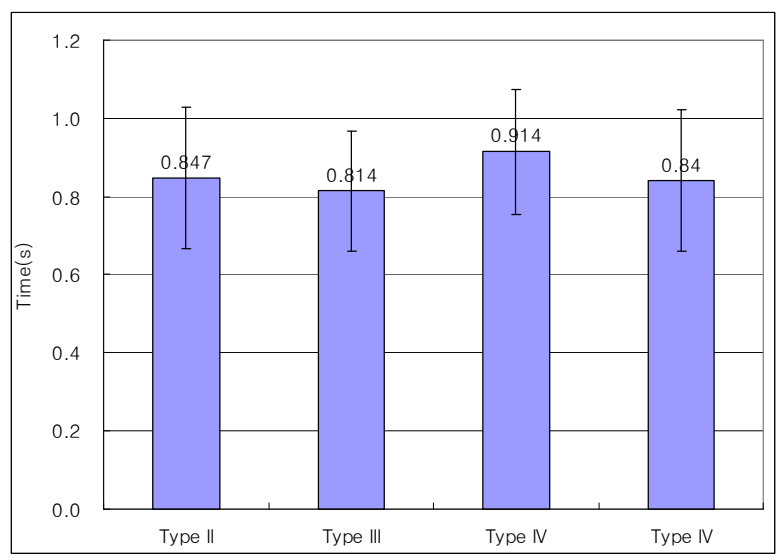

Figure 3. Reaction times depending upon burner-control arrangement type

\subsection{Effect of subjects' gender}

피실험자의 성별에 따른 평균 반응시간은 Figure 4에 나 와 있다. 반응시간은 남성에 비하여 여성이 작았으나 분산 분석, Duncan의 다중범위 검정에서 유의수준 $5 \%$ 에서 통계 적으로 유의한 차이가 없는 것으로 나타났다.

\subsection{Comparison with previous studies}

현재까지 수행되어 온 가스레인지 버너-조종장치 연결 형 태에 대한 스테레오타이프 연구 결과가 Table 3에 정리되어 있다. 설문조사를 이용한 연구(Hsu and Peng, 1993; Kee, 
2011a, b; Shinar and Acton, 1978; Wu, 1997)에서는 Type III가 가스레인지에 대한 스테레오타이프로 제시되었 다. 나무 혹은 아크릴 수지 (perspex)로 만든 모형을 이용 한 연구(Chapanis and Lindenbaum, 1959; Ray and Ray, 1979)에서는 Type II가, Wu(1997)의 연구에서는 Type V 가 가스레인지 조작 시 에러율이 가장 낮거나 반응시간이 가 장 짧았다. 컴퓨터 시뮬레이션을 이용한 Hsu와 Peng (1993) 은 Type III를 스테레오타이프로 제시하였다. 실물 가스레인 지를 사용한 본 연구의 결과인 Type III는 모형을 이용한 연 구 결과와는 달랐으나, 설문조사를 이용한 연구와 결과가 일 치하였다.

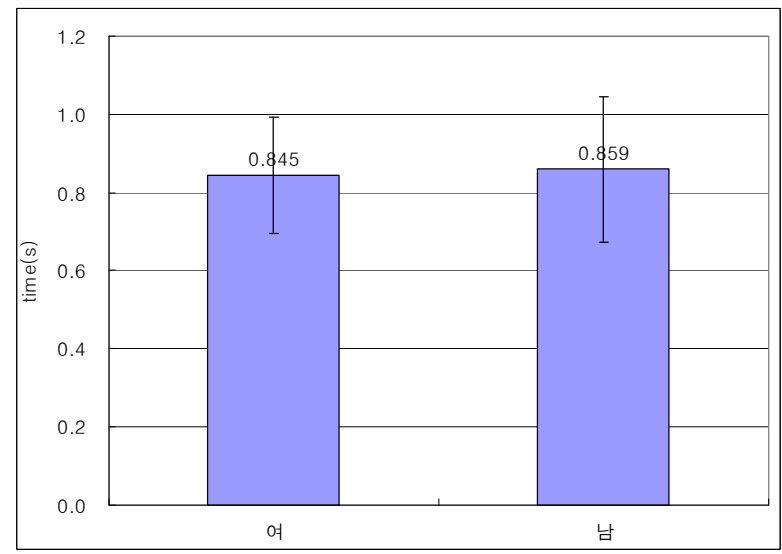

Figure 4. Reaction times depending upon subjects' gender

Table 3. Results of this study and previous studies

\begin{tabular}{c|c|c|c|c|c|c|c}
\hline & $\begin{array}{c}\text { This } \\
\text { study }\end{array}$ & $\begin{array}{c}\text { Chapanis \& } \\
\text { Lindenbaum }^{1}\end{array}$ & $\begin{array}{c}\text { Ray \& } \\
\text { Ray }^{1}\end{array}$ & $\begin{array}{c}\text { Shinar \& } \\
\text { Acton }^{2}\end{array}$ & $\begin{array}{c}\text { Hsu \& } \\
\text { Peng }^{3}\end{array}$ & Wu & Kee $^{2}$ \\
\hline $\begin{array}{c}\text { Streo- } \\
\text { type }\end{array}$ & III & II & II & III & III & $\begin{array}{c}\text { III }^{2} \\
\text {, }\end{array}$ & III \\
\hline
\end{tabular}

1: physical model; 2: questionnaire survey; 3 : questionnaire survey and computer simulation

\section{Discussion and Conclusion}

본 연구에서는 실물 가스레인지를 사용한 실험을 통하여 버너-조종장치 연결 형태에 대한 한국인의 스테레오타이 프로 Type III를 제시하였다. 설문조사를 이용한 스테레오타 이프에 대한 연구가 대부분의 상황, 특히 3차원 조종장치표시장치 배열에서 부적절하다고 알려져 있다(Hoffmann, 2009). 따라서 3차원인 가스레인지를 대상으로 설문조사나 모형이 아닌 실물을 처음으로 사용한 본 연구의 결과가 기존
연구에 비하여 좀 더 신뢰성이 높을 것으로 기대된다.

분산분석 및 Duncan의 다중범위 검정에서 성별이 버너조종장치 가스레인지 연결 형태별 반응시간에 유의한 영향 을 미치지 않는 것으로 나타났다(Table 2, Figure 4). 일반 적으로 남성에 비하여 여성의 가스레인지 사용 경험이 많아 가스레인지 조작시간도 여성이 작을 것으로 추정되나, 본 연 구의 실험에서 반응시간에는 성별 간 통계적 유의차가 나타 나지 않았다. 스테레오타이프가 사용 경험이나 사전 지식에 영향을 받지 않고 초기 직관에 의해 조사되어야 함을 고려할 때 (Shinar and Acton, 1978), 여성의 사전 사용 경험이나 지식의 유의한 영향이 나타나지 않은 본 연구는 실험 설계 및 피실험자 선정에 문제가 없었음을 나타내는 것이라 할 수 있다.

가스레인지 스테레오타이프 조사 방법 즉, 설문조사, 모형 혹은 컴퓨터 시뮬레이션 중 어떤 방법을 이용하는가에 따라 연구 결과가 달리 나타났다(Table 3). 특히, 같은 중국인에 대한 스테레오타이를 조사한 Wu(1997)의 연구에서는 설문 조사 결과와 모형을 이용한 연구 결과가 달라 연구 방법의 선택이 중요함을 보였다. 이러한 기존 연구 결과를 살펴볼 때 한국인에 대한 설문조사에 바탕한 연구는 있으나, 모형을 이용한 연구가 없는 상황에서 실물 가스레인지를 사용한 본 연구가 필요하고 또 그 의의가 있다 하겠다.

실물 가스레인지를 이용한 본 연구의 결과가 설문조사에 근거한 한국인을 대상으로 한 전 연구(Kee, 2011a, b) 와 일치하였다. 이는 연구 방법에 따라 가스레인지 스테레오타 이프에 차이를 보인 기존 연구와 차이를 보인 것이라 할 수 있다. 연구 방법에 따른 차이의 원인으로는 설문조사를 이용 한 기존 연구에서는 Figure 2에서 보는 바와 같이 피실험자 에게 제시한 가스레인지 그림이 단순한 선과 원으로 구성되 어 있어 현실성이 떨어진 점을 들 수 있다. 이러한 추정은 실물에 가까운 그림(Figure 5)을 제시한 설문조사에 근거한 $\mathrm{Kee}(2011 \mathrm{a}, \mathrm{b})$ 와 실물을 사용한 본 연구의 결과가 일치하 는 데서 입증될 수 있다. 이와 같은 추정은 많은 경우 설문 조사와 하드웨어를 이용한 스테레오타이프 연구 결과가 같 다는 Hoffmann(1997)의 연구와도 일치한다. 따라서 설문

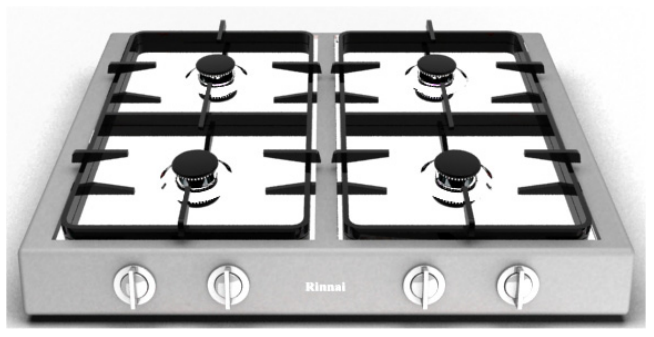

Figure 5. Illustration of gas range used in $\operatorname{Kee}(2011 \mathrm{a}, \mathrm{b})$ 
조사를 이용한 스테레오타이프 조사 연구에서는 가능하면 조사 대상의 실물에 가까운 그림이나 사진을 제시하여야 한 다. 그러나 이러한 추정에 대해서는 실물과의 근접성 정도에 따른 스테레오타이프 연구 결과의 차이에 대한 추후 연구가 있을 때까지는 조심스러운 접근이 필요할 것으로 생각된다.

본 연구는 20대 전후 대학생만을 대상으로 실험을 수행하 였으나, 좀 더 정확한 가스레인지 스테레오타이를 조사하기 위해서는 다양한 연령층에 대한 추후 연구가 필요하다.

\section{References}

Chapanis, A. and Lindenbaum, L. E., A reaction-time study of four control-display linkage, Human Factors, 1, 1-7, 1959.

Hoffmann, E. R., Strength of component principles determining direction of turn stereotypes-linear displays with rotary controls, Ergonomics, 40(2), 199-222, 1997.

Hoffmann, E. R., Do paper-and-pencil tests give an accurate measure of stereotype strength?: A review of available data, International Journal of Industrial Ergonomics, 39, 904-912, 2009.

Hsu, S-H. and Peng, Y., Control/display relationship of the four-burner stove: A reexamination, Human Factors, 35(4), 745-749, 1993.

Kee, D., Stereotype for control-display relationship o four-burner range for Korean, Journal of the Ergonomics Society of Korea, 30(3), 389-394, 2011a.

Kee, D., Stereotype and effective cues for burner-control relationship of four-stove range, Journal of the Korean Institute of Industrial Engineers, 37(2), 118-123, 2011b.

Kee, D., Lee, K. T., Park, J. H. and Cho, K. I., Ergonomics, Hankyungsa, Seoul, 2010.
Kromer, K. H. E., Kroemer, H. B. and Kroemer-Elbert, K. E., Ergonomics: How to design for ease and efficiency, Prentice-Hall, Inc., London, 1994.

Ray, R. D. and Ray, W. D., An analysis of domestic cooker control design, Ergonomics, 22, 1243-1248, 1979.

Shinar, D. and Acton, M. B., Control-display relationships on the fourburner range: Population stereotypes versus standard, Human Factors, 20, 13-17, 1978.

Wu, S-P., Further studies on the spatial compatibility of four control-display linkages, International Journal of Industrial Ergonomics, 19, 353-360, 1997.

\section{Author listings}

Doyung Kee: dhkee@kmu.ac.kr

Highest degree: Ph.D., Department of Industrial Engineering, POSTECH Position title: Professor, Department of Industrial and Management Engineering, Keimyung University

Areas of interest: Population stereotype, product design, posture classification scheme, industrial safety, Musculoskeletal disorders

Date Received : 2012-03-20

Date Revised :2012-05-22

Date Accepted : 2012-05-22 